\title{
Single-particle cryo-EM at atomic resolution
}

 \\ ${ }^{1}$ MRC Laboratory of Molecular Biology, Cambridge, ${ }^{2}$ UK, Materials and Structural Analysis \\ Division, Thermo Fisher Scientific, Eindhoven, The Netherlands \\ asente@mrc-Imb.cam.ac.uk
}

The three-dimensional positions of atoms in protein molecules define their structure and the roles they perform in biological processes. The more precisely atomic coordinates are determined, the more chemical information can be derived and the more mechanistic insights into protein function may be inferred. With breakthroughs in electron detection and image processing technology, electron cryo-microscopy (cryo-EM) single-particle analysis has yielded protein structures with increasing levels of detail in recent years. However, obtaining cryo-EM reconstructions with sufficient resolution to visualise individual atoms in proteins has thus far been elusive. We recently demonstrated how a combination of new algorithms (Bayesian polishing, optical aberration correction and Ewald sphere correction) and hardware (a new cold field emission gun, new energy filter and the Falcon 4 detector) yields a $1.7 \AA$ resolution cryo-EM reconstruction for a prototypical human membrane protein, the 3 GABAA receptor homopentamer. Such maps allow a detailed understanding of small molecule coordination, visualisation of solvent molecules and alternative conformations for multiple amino acids, as well as unambiguous building of ordered acidic side chains and glycans. Moreover, the scattering potential from many hydrogen atoms can be visualised in difference maps, allowing a direct analysis of hydrogen bonding networks. Applied to mouse apoferritin, our strategy led to a $1.2 \AA$ resolution reconstruction that, for the first time, offers a genuine atomic resolution view of a protein molecule using single particle cryo-EM.

Acta Cryst. (2020). A76, a220 